PROCEEDINGS OF THE

AMERICAN MATHEMATICAL SOCIETY

Volume 126, Number 1, January 1998, Pages 35-43

S 0002-9939(98)04464-5

\title{
AMPLE AND SPANNED VECTOR BUNDLES OF TOP CHERN NUMBER TWO ON SMOOTH PROJECTIVE VARIETIES
}

\author{
ATSUSHI NOMA
}

(Communicated by Ron Donagi)

\begin{abstract}
The purpose of this paper is to classify ample and spanned vector bundles of top Chern number two on smooth projective varieties of arbitrary dimension defined over an algebraically closed field of characteristic zero.
\end{abstract}

\section{INTRODUCTION}

We work over an algebraically closed field $k$ of characteristic zero.

The purpose here is to classify ample and spanned vector bundles of top Chern number two on smooth projective varieties of arbitrary dimension. Here we recall that a vector bundle $E$ is ample if the tautological line bundle $\mathcal{O}_{\mathbb{P}(E)}(1)$ of the projective space bundle $\mathbb{P}(E)$ is ample in usual sense, and that $E$ is spanned if $E$ is generated by its global sections.

There are several studies on the problem of classifying ample and spanned vector bundles $E$ of rank $r \geq n$ with small top Chern number $c_{n}(E)$ on normal projective varieties $X$ of dimension $n$. In case $c_{n}(E)=1$, Lanteri and Sommese [11] have shown that $(X, E) \cong\left(\mathbb{P}^{n}, \mathcal{O}_{\mathbb{P}^{n}}(1)^{\oplus n}\right)$ when $X$ are normal surfaces or Gorenstein 3 -folds with only isolated singularities. In the higher dimensional case, Wiśniewski [15] has shown that the same is true when $X$ are smooth projective varieties. Fujita has pointed out that the same result for projective varieties $X$ with only log terminal singularities also follows from Zhang's result [17] together with the Lanteri-Sommese argument (see Proposition 5.1 below). In case $c_{n}(E)=2$, the structure of $(X, E)$ has been described explicitly when $X$ are smooth surfaces ([13], see also [10]). This result has been extended to the case when $X$ are normal Gorenstein surfaces ([14]).

Our main result is the following:

Theorem 1.1. Let $X$ be a smooth projective variety of dimension $n \geq 2$, and $E$ an ample and spanned vector bundle with rank $E=r \geq n$ and $c_{n}(E)=2$ on $X$. Then $(X, E)$ is one of the following:

(1) $X$ has a finite morphism $f: X \rightarrow \mathbb{P}^{n}$ of degree 2 over a projective space $\mathbb{P}^{n}$, and $E \cong f^{*} \mathcal{O}_{\mathbb{P} n}(1)^{\oplus n}$.

(2) $(X, E) \cong\left(\mathbb{P}^{n}, \mathcal{O}_{\mathbb{P}^{n}}(2) \oplus \mathcal{O}_{\mathbb{P}^{n}}(1)^{\oplus n-1}\right)$.

Received by the editors April 29, 1996.

1991 Mathematics Subject Classification. Primary 14F05, 14J60; Secondary 14C05.

Key words and phrases. ample vector bundle, spanned vector bundle, zero cycle, adjunction map.

(C)1998 American Mathematical Society 
(3) $(X, E) \cong\left(\mathbb{Q}^{n}, \mathcal{O}_{\mathbb{Q}^{n}}(1)^{\oplus n}\right)$, where $\mathbb{Q}^{n}$ is a smooth quadric hypersurface in $\mathbb{P}^{n+1}$, and $\mathcal{O}_{\mathbb{Q}^{n}}(1)$ is the hyperplane line bundle.

(4) $X$ is isomorphic to a projective space bundle $\mathbb{P}_{C}(\mathcal{F})$ over an elliptic curve $C$ with the projection $\pi: \mathbb{P}_{C}(\mathcal{F}) \rightarrow C$ and with the tautological line bundle $\mathcal{O}_{\mathbb{P}_{C}(\mathcal{F})}(1)$, and $E \cong \pi^{*} \mathcal{E} \otimes \mathcal{O}_{\mathbb{P}_{C}(\mathcal{F})}(1)$. Here $\mathcal{F}$ and $\mathcal{E}$ are indecomposable vector bundles of rank $n$ and degree 1 on $C$.

(5) $X$ is isomorphic to a projective space bundle $\mathbb{P}_{C}(\mathcal{F})$ over a hyperelliptic curve $C$ of genus $g \geq 2$ with the projection $\pi: \mathbb{P}_{C}(\mathcal{F}) \rightarrow C$ and with the tautological line bundle $\mathcal{O}_{\mathbb{P}_{C}(\mathcal{F})}(1)$, and $E \cong \pi^{*} \mathcal{E} \otimes \mathcal{O}_{\mathbb{P}_{C}(\mathcal{F})}(1)$. Here $\mathcal{F}$ and $\mathcal{E}$ are vector bundles of rank $n$ on $C$ such that the system $|\operatorname{det} \mathcal{F} \otimes \operatorname{det} \mathcal{E}|$ is the hyperelliptic pencil $g_{2}^{1}$.

Conversely, every bundle in the all cases but (5) is ample and spanned with $c_{n}(E)=$ 2 .

Remark 1.2. When $n=2$, the case (5) is void (see [13, Theorem 6.1 and Proposition $5.4]$ ). When $n \geq 3$, I do not know whether a bundle in the case (5) exists or not.

Our proof proceeds as follows. The essential case of the proof is $\operatorname{rank} E=\operatorname{dim} X$ $(\S 2)$. In this case, an invariant $\operatorname{sp}(E)$ of Ballico [2] divides our situation into two subcases: $\operatorname{sp}(E)=n$ and $\operatorname{sp}(E)>n(\S 3)$. When $\operatorname{sp}(E)=n$, Ballico's theorem [2] and Zhang's theorem [17] together with the Lanteri-Sommese argument [11] imply the precise structure $(\S \S 4,5)$. When $\operatorname{sp}(E)>n$, the key step is to show that the adjoint bundle $K_{X} \otimes \operatorname{det} E$ fails to be ample $(\S \S 6,7)$. Then Fujita's theorem [6] tells us the structure of $(X, E)$, and we pick up the bundles in our case $(\S 8)$.

I would like to express my gratitude to Professor Takao Fujita, who told me the result of Zhang and its application to this study in $\S 5$. Also I am grateful to the referee of [14], who gave me invaluable advice for the case $\operatorname{sp}(E)>n$.

\section{CASE $\operatorname{rank} E>\operatorname{dim} X$}

Once we obtain Theorem 1.1 for $r=n$, it turns out that there is no bundle when $r>n$, by using a standard argument (see [10], [14]): If $r>n$, by Serre's lemma, we have an exact sequence of vector bundles

$$
0 \rightarrow \mathcal{O}_{X}^{\oplus r-n} \rightarrow E \rightarrow E^{\prime} \rightarrow 0,
$$

since $E$ is spanned. Then $E^{\prime}$ is an ample and spanned vector bundle of rank $n$, and $c_{n}(E)=2$. By Theorem 1.1 for $r=n$, we easily check in each case that

$$
H^{1}\left(X, E^{\prime \vee}\right)=0 .
$$

Indeed, for (1), this follows from the facts that $f_{*} \mathcal{O}_{X}$ and hence $f_{*} E^{\vee}$ are direct sums of line bundles on $\mathbb{P}^{n}$ and that $H^{1}\left(X, E^{\vee}\right)=H^{1}\left(\mathbb{P}^{n}, f_{*} E^{\vee}\right)$; for (2) and (3), this is clear; for (4) and (5), this follows from the facts that $E$ is the extension of a line bundle of the form $\mathcal{O}_{\mathbb{P}_{C}(\mathcal{F})}(1) \otimes \pi^{*} \mathcal{M}$ for some $\mathcal{M} \in \operatorname{Pic} C$ by line bundles of the same type and that $H^{1}\left(\mathbb{P}_{C}(\mathcal{F}), \mathcal{O}_{\mathbb{P}_{C}(\mathcal{F})}(-1) \otimes \pi^{*} \mathcal{M}^{\vee}\right)=0$. Therefore the exact sequence above is split in each case. This contradicts the ampleness of $E$.

\section{Notation And Ballico's invariant}

From now on, we assume that $r=n$. Let us start with some notation in a more general setting, and recall the definition of the invariant $\operatorname{sp}(E)$ of Ballico [2] (see also [13]). 
Let $X$ be a normal projective variety of dimension $n$, and $E$ a spanned vector bundle of rank $n$ with $c_{n}(E)>0$ on $X$. By $|E|$ we denote $\mathbb{P}\left(H^{0}(X, E)^{\vee}\right)=$ $\left(H^{0}(X, E) \backslash\{0\}\right) / k^{*}$, and $[t]$ is the point of $|E|$ corresponding to $t \neq 0 \in H^{0}(X, E)$.

We consider the family of zeros $F=\{([t], p) \in|E| \times X ; t(p)=0\}$, a projective space bundle $\mathbb{P}_{X}\left(\mathcal{F}_{E}\right)$ associated with a vector bundle $\mathcal{F}_{E}$ whose dual bundle is the kernel of the evaluation map ev $: H^{0}(X, E) \otimes \mathcal{O}_{X} \rightarrow E$. Hence we get the following diagram:

$$
\begin{array}{ccc}
|E| \times X \quad & \supset & \stackrel{\Psi}{\rightarrow} \quad X \\
& \Phi \downarrow \\
& |E| .
\end{array}
$$

Here $\Phi: F \rightarrow|E|$ and $\Psi: F \rightarrow X$ denote the first and second projections. Note that the fibre $F_{\{p\}}:=\Psi^{-1}(p)$ of $\Psi$ over a closed point $p \in X$ is $\left|E \otimes \mathcal{I}_{\{p\}}\right| \times\{p\}$, where we set $\left|E \otimes \mathcal{I}_{\{p\}}\right|=\mathbb{P}\left(H^{0}\left(X, E \otimes \mathcal{I}_{\{p\}}\right)^{\vee}\right)$. On the other hand, $\Phi$ is a finite flat morphism of degree $c_{n}(E)$ over a dense and open subset

$$
|E|_{\text {reg }}:=\left\{[t] \in|E| ; \operatorname{dim}(t)_{0}=0 \text { and }(t)_{0} \text { is on the Cohen-Macaulay locus of } X\right\} \text {. }
$$

So we have a morphism $\tau:|E|_{\text {reg }} \rightarrow \operatorname{Hilb}_{X}^{c_{n}(E)}$ by the universality of the Hilbert scheme $\operatorname{Hilb}_{X}^{c_{n}(E)}$ of $c_{n}(E)$ points in $X$. By considering the norm morphism $\nu$ from $\operatorname{Hilb}_{X}^{c_{n}(E)}$ to the $c_{n}(E)$ th symmetric product $S^{c_{n}(E)}(X)$ of $X$, we also have a morphism $\rho=\nu \circ \tau:|E|_{\mathrm{reg}} \rightarrow S^{c_{n}(E)}(X)$.

Then we define $\operatorname{sp}(E)$ by

$$
\operatorname{sp}(E)=\operatorname{dim} \tau\left(|E|_{\mathrm{reg}}\right) .
$$

Since $\nu$ is isomorphic over the open subset parameterizing distinct smooth $c_{n}(E)$ points of $X$, we have $\operatorname{sp}(E)=\operatorname{dim} \rho\left(|E|_{\text {reg }}\right)$. Hence this invariant is Ballico's in [2]. By definition, we have $n \leq \operatorname{sp}(E) \leq n \cdot c_{n}(E)$.

By [13, Proposition 1.1], for every $[t] \in|E|_{\mathrm{reg}}$, the fibre $\tau^{-1}(\tau([t]))$ of $\tau$ over $\tau([t])$ is $\left|E \otimes \mathcal{I}_{(t)_{0}}\right| \cap|E|_{\text {reg }}$, where $\left|E \otimes \mathcal{I}_{(t)_{0}}\right|:=\mathbb{P}\left(H^{0}\left(X, E \otimes \mathcal{I}_{(t)_{0}}\right)^{\vee}\right) \subseteq|E|$. Hence, for a general section $t \in H^{0}(X, E)$, it holds that

$$
\operatorname{sp}(E)=\operatorname{dim}_{k} H^{0}(X, E)-\operatorname{dim}_{k} H^{0}\left(X, E \otimes \mathcal{I}_{(t)_{0}}\right) .
$$

In particular, the subset

$$
|E|_{\text {comp }}:=\left\{[t] \in|E|_{\text {reg }} ;(t)_{0} \text { is smooth and } t \text { satisfies }(3.2)\right\}
$$

is dense and open in $|E|$.

$$
\text { 4. CASE } \operatorname{sp}(E)=\operatorname{dim} X
$$

We recall the structure theorem on $(X, E)$ of $\operatorname{sp}(E)=n$, due to Ballico [2]. Here we give a proof by using the formula (3.2).

Proposition 4.1. (Ballico [2, Theorem 4.1]) Let $E$ be an ample and spanned vector bundle of rank $n$ on a normal projective variety $X$ of dimension $n$. Assume that $\operatorname{sp}(E)=n$. Then there exist an ample and spanned vector bundle $E^{\prime}$ with $\operatorname{rank} E^{\prime}=$ $n$ and $c_{n}\left(E^{\prime}\right)=1$ on a normal projective variety $X^{\prime}$ with $\operatorname{dim} X^{\prime}=n$, and a finite morphism $f: X \rightarrow X^{\prime}$ of degree $c_{n}(E)$ such that $E \cong f^{*}\left(E^{\prime}\right)$. 
Proof. Consider a morphism $\gamma: X \rightarrow \mathbb{G}:=\operatorname{Grass}\left(H^{0}(X, E), n\right)$ from $X$ to the Grassmann variety of $n$-quotients associated with the evaluation map $H^{0}(X, E) \otimes$ $\mathcal{O}_{X} \rightarrow E$. Let $X^{\prime}$ be the normalization of the image $\gamma(X)$ with the induced morphism $f: X \rightarrow X^{\prime}$. Let $E^{\prime}$ be the pull-back of the universal quotient bundle on $\mathbb{G}$ onto $X^{\prime}$, so $E \cong f^{*}\left(E^{\prime}\right)$ and $E^{\prime}$ is spanned. Since $E$ is ample, $f$ is finite; hence $E^{\prime}$ is also ample. For $[t] \in|E|_{\text {comp }}$ and $p \in(t)_{0}$, we have $H^{0}\left(X, E \otimes \mathcal{I}_{(t)_{0}}\right)=H^{0}\left(X, E \otimes \mathcal{I}_{\{p\}}\right)$ as a subspace of $H^{0}(X, E)$ by assumption on $\operatorname{sp}(E)$ and (3.2). By definition of $\gamma$, every point of $(t)_{0}$ is mapped to the same point by $\gamma$, and hence $\operatorname{deg}(f) \geq c_{n}(E)$. Since $c_{n}(E)=(\operatorname{deg} f) \cdot c_{n}\left(E^{\prime}\right)$, by the positivity of $c_{n}\left(E^{\prime}\right)$, we have $\operatorname{deg}(f)=c_{n}(E)$ and $c_{n}\left(E^{\prime}\right)=1$, as required.

\section{Proof of the theorem When $\operatorname{sp}(E)=\operatorname{dim} X$}

If the pair $(X, E)$ in Theorem 1.1 has $\operatorname{sp}(E)=n$, by Proposition 4.1, $X$ has a double cover $f: X \rightarrow X^{\prime}$ over a normal projective variety $X^{\prime}$ of dimension $n$, and $E$ is the pull-back of an ample and spanned vector bundle $E^{\prime}$ of $c_{n}\left(E^{\prime}\right)=1$ on $X^{\prime}$. Since $X$ and $X^{\prime}$ are normal and $\operatorname{deg} f=2$, there exists an involution $\iota$ on $X$ such that the quotient $X /\langle\iota\rangle$ is $X^{\prime}$. By [9, Proposition 1.7], $X^{\prime}$ has only log terminal singularities, since $X$ is smooth. Thus by the following proposition, which is a consequence of Zhang's Theorem [17, Theorem 1] (see also [16, Theorem 3]), we have $X^{\prime} \cong \mathbb{P}^{n}$ and $E^{\prime} \cong \mathcal{O}_{\mathbb{P}^{n}}(1)^{\oplus n}$; this is the case (1).

Proposition 5.1 (Pointed out by Fujita). Let $X$ be a projective variety of dimension $n$ with only log terminal singularities, and $E$ an ample and spanned vector bundle with $\operatorname{rank} E=n$ and $c_{n}(E)=1$ on $X$. Then $(X, E) \cong\left(\mathbb{P}^{n}, \mathcal{O}_{\mathbb{P}^{n}}(1)^{\oplus n}\right)$.

Proof. According to the Lanteri-Sommese argument [11], we consider the diagram (3.1) for $(X, E)$. Set $N=\operatorname{dim}|E|$. Since $\Phi$ is generically one-to-one by assumption

and $\Psi$ is dominant, if $\ell$ is a general line in $|E|$, then $C:=\Phi^{-1}(\ell) \cong \ell$ and $\Psi(C)$ is a curve. Since $\Phi^{*} \mathcal{O}_{|E|}(1)$ is isomorphic to the tautological line bundle $\mathcal{O}_{F}(1):=$ $\mathcal{O}_{\mathbb{P}\left(\mathcal{F}_{E}\right)}(1)$ of $F=\mathbb{P}_{X}\left(\mathcal{F}_{E}\right)$, the curve $C$ is the complete intersection of divisors associated with $\mathcal{O}_{F}(1)$. On the other hand, we have $K_{F} \cong \Psi^{*}\left(K_{X} \otimes \operatorname{det} E\right) \otimes$ $\mathcal{O}_{F}(-N+n-1)$. Therefore, by the adjunction formula for $C$, we have

$$
\begin{aligned}
-2=\left(K_{F} \otimes \mathcal{O}_{F}(N-1), C\right) & =\left(\Psi^{*}\left(K_{X} \otimes \operatorname{det} E\right) \otimes \mathcal{O}_{F}(n-2), C\right) \\
& =\left(\Psi^{*}\left(K_{X} \otimes \operatorname{det} E\right), C\right)+n-2 .
\end{aligned}
$$

Hence $\left(K_{X} \otimes \operatorname{det} E, \Psi_{*} C\right)=-n$ by the projection formula. Therefore, by Zhang's Theorem [17, Theorem 1], we have $(X, E) \cong\left(\mathbb{P}^{n}, \mathcal{O}_{\mathbb{P}^{n}}(1)^{\oplus n}\right)$.

\section{CASe $\operatorname{sp}(E)>\operatorname{dim} X$; FIRST STEP}

Lemma 6.1. Let $X$ be a normal projective variety of dimension $n$, and $E$ a spanned vector bundle with $\operatorname{rank} E=n$ and $c_{n}(E)=2$ on $X$. Set

$$
\begin{aligned}
\mathcal{V} & =\left\{(p, q) \in X^{2} ; p \neq q, t(p)=t(q)=0 \text { for some }[t] \in|E|_{\mathrm{comp}}\right\}, \\
F^{\prime} & =F \cap\left(|E|_{\mathrm{comp}} \times X\right) .
\end{aligned}
$$

Then $\mathcal{V}$ is a locally closed subvariety of dimension $\operatorname{sp}(E)$, and

$$
\eta: F^{\prime} \rightarrow \mathcal{V}, \quad([t], p) \mapsto(p, q) \quad\left((t)_{0}=\{p, q\}\right),
$$


is a morphism over $X$. By base change, we have a surjection

$$
\begin{aligned}
\eta_{\{p\}}: F_{\{p\}}^{\prime}= & \left(\left|E \otimes \mathcal{I}_{\{p\}}\right| \cap|E|_{\text {comp }}\right) \times\{p\} \rightarrow \mathcal{V}_{\{p\}}, \\
& ([t], p) \mapsto q \quad\left((t)_{0}=\{p, q\}\right) .
\end{aligned}
$$

Here $\mathcal{V}_{\{p\}}$ is the fibre of the first projection from $\mathcal{V}$ to $X$ over $p \in X$. In particular, $X$ is covered with rational varieties of dimension $\operatorname{sp}(E)-n$.

Proof. Consider the following incidence correspondence:

$$
\begin{gathered}
G=\left\{([t], p, q) \in|E| \times X^{2} ; t(p)=t(q)=0\right\}, \\
\mu: G \longrightarrow F, \quad([t], p, q) \mapsto([t], p) .
\end{gathered}
$$

As a scheme, $G$ is the projective space bundle $\mathbb{P}_{X^{2}}\left(\mathcal{G}_{E}\right)$ associated with the cokernel $\mathcal{G}_{E}$ of $p_{1}^{*}\left(\mathrm{ev}^{\vee}\right) \oplus p_{2}^{*}\left(\mathrm{ev}^{\vee}\right): p_{1}^{*}\left(E^{\vee}\right) \oplus p_{2}^{*}\left(E^{\vee}\right) \rightarrow H^{0}(E)^{\vee} \otimes \mathcal{O}_{X^{2}}$, where $p_{i}: X^{2} \rightarrow$ $X(i=1,2)$ are the first and second projections. And $\mu: G=\mathbb{P}_{X^{2}}\left(\mathcal{G}_{E}\right) \hookrightarrow$ $\mathbb{P}_{X^{2}}\left(p_{1}^{*} \mathcal{F}_{E}\right)=\mathbb{P}_{X}\left(\mathcal{F}_{E}\right) \times_{X} X^{2} \rightarrow \mathbb{P}_{X}\left(\mathcal{F}_{E}\right)=F$ is induced by a natural surjection $p_{1}^{*} \mathcal{F}_{E} \rightarrow \mathcal{G}_{E}$. Let $\psi: G \rightarrow X^{2}$ be the projection. Set $G^{\prime}=G \cap\left(|E|_{\operatorname{comp}} \times\left(X^{2} \backslash \Delta\right)\right)$, where $\Delta$ is the diagonal of $X^{2}$. Let $\mu^{\prime}: G^{\prime} \rightarrow F^{\prime}$ be the induced morphism from $\mu$.

Then $\mu^{\prime}$ is an isomorphism: Indeed, the fibre of $\mu$ over $([t], p) \in F^{\prime}$ is schemetheoretically 2 distinct points, $([t], p, p)$ and $([t], p, q)$ with $(t)_{0}=\{p, q\}$. On the other hand, $\mu$ is projective. Hence $\mu: \mu^{-1}\left(F^{\prime}\right) \rightarrow F^{\prime}$ is a finite, flat, unramified morphism of degree 2. Since $\mu^{\prime}$ is one-to-one and $G^{\prime}$ is an open subset of $\mu^{-1}\left(F^{\prime}\right)$, the morphism $\mu^{\prime}$ is isomorphic.

Since $\mathcal{V}$ is the image $\psi \circ \mu^{\prime-1}\left(F^{\prime}\right), \mathcal{V}$ is irreducible and locally closed. We set $\eta=\psi \circ \mu^{\prime-1}$; we have the morphism $\eta$. Since $\operatorname{sp}(E)$ is the dimension of the quotient of $\mathcal{V}$ by the natural involution, we have $\operatorname{dim} \mathcal{V}=\operatorname{sp}(E)$. By base change, we have $\eta_{\{p\}}$. Let $p \in X$ be a general closed point so that $\mathcal{V}_{\{p\}}$ is of dimension $\operatorname{sp}(E)-n$. Since every fibre of $\eta_{\{p\}}$ over $q \in \mathcal{V}_{\{p\}}$ is $\left(\left|E \otimes \mathcal{I}_{\{p, q\}}\right| \cap|E|_{\text {comp }}\right) \times\{p\}$, $\eta_{\{p\}}$ induces a generically isomorphism between $\mathcal{V}_{\{p\}}$ and a general $(\operatorname{sp}(E)-n)$ plane in $\left|E \otimes \mathcal{I}_{\{p\}}\right|$. Hence $\mathcal{V}_{\{p\}}$ is rational. Since $F^{\prime}$ dominates $X, \mathcal{V}$ dominates $X$ via the first projection, hence via the second projection by symmetry. Thus $X$ is covered with rational varieties $\mathcal{V}_{\{p\}}$ of dimension $\operatorname{sp}(E)-n$.

\section{CASE $\operatorname{sp}(E)>\operatorname{dim} X ; \operatorname{KEY} \operatorname{sTEP}$}

Proposition 7.1. Let $X$ be a normal projective variety of dimension $n$ and $E$ a spanned vector bundle with rank $E=n$ and $c_{n}(E)=2$ on $X$. Assume that $\operatorname{sp}(E)>n$ and that $X$ is Gorenstein. Then there exists a projective integral curve $C$ on $X$ such that $\left(K_{X} \otimes \operatorname{det} E, C\right) \leq 0$. In particular, $K_{X} \otimes \operatorname{det} E$ is not ample.

Proof. Consider the diagram (3.1) for $(X, E)$. Since $\operatorname{dim} F=\operatorname{dim}|E|$, a closed subset $B:=\left\{[t] \in|E| ; \operatorname{dim} \Phi^{-1}([t])=\operatorname{dim}(t)_{0} \geq 1\right\}$ has codimension at least 2 in $|E|$. Over the outside of $R:=\Phi^{-1}(B), \Phi$ is finite of degree 2 ; hence there exists an involution $\iota: F \backslash R \rightarrow F \backslash R$. We extend $\iota$ to the morphism $\tilde{\iota}: F \backslash Z \rightarrow F$ with the closed subset $Z \subset F$ of codimension at least 2, by using the valuative criterion of properness. So we have an injective map $\mathcal{O}_{F \backslash Z}\left(\tilde{\iota}^{*} K_{F}\right) \rightarrow \mathcal{O}_{F \backslash Z}\left(K_{F}\right)$ of sheaves on $F \backslash Z$, where $\tilde{\iota}^{*} K_{F}$ is the pull-back of the canonical divisor $K_{F}$ of $F$ and $\mathcal{O}_{F \backslash Z}\left(\tilde{\iota}^{*} K_{F}\right)$ is a line bundle on $F \backslash Z$ associated with $\tilde{\iota}^{*} K_{F}$.

Let $p \in X$ be a general closed point so that a closed subset $Z \cap F_{\{p\}}$ has codimension at least 2 in the fibre $F_{\{p\}}=\left|E \otimes \mathcal{I}_{\{p\}}\right| \times\{p\}$ of $\Psi$ over $p$; the closure of the 
support of $\mathcal{O}_{F \backslash Z}\left(K_{F}\right) / \mathcal{O}_{F \backslash Z}\left(\widetilde{\iota}^{*} K_{F}\right)$ has codimension at least 1 in $F_{\{p\}}$; and $\mathcal{V}_{\{p\}}$ has dimension $\operatorname{sp}(E)-n$ (see Lemma 6.1). Let $\ell$ be a general line in $\left|E \otimes \mathcal{I}_{\{p\}}\right|$ so that

(1) $Z \cap(\ell \times\{p\})=\emptyset$;

(2) $\ell \times\{p\} \not \subset \overline{\operatorname{Supp}\left(\mathcal{O}_{F \backslash Z}\left(K_{F}\right) / \mathcal{O}_{F \backslash Z}\left(\tilde{\iota}^{*} K_{F}\right)\right)}$;

(3) $\ell \times\{p\}$ is not in the fibre of $\eta_{\{p\}}$ in (6.1.2).

Here ${ }^{-}$denotes the closure in $F$. By $(1), \tilde{\ell}:=\tilde{\iota}(\ell \times\{p\})$ is a complete curve on $F$ and the intersection number $\left(\mathcal{O}_{F \backslash Z}\left(\tilde{\iota}^{*} K_{F}\right), \ell \times\{p\}\right)$ is defined. By the projection formula and (2), we have

$$
\left(K_{F}, \tilde{\ell}\right)=\left(\mathcal{O}_{F \backslash Z}\left(\tilde{\iota}^{*} K_{F}\right), \ell \times\{p\}\right) \leq\left(K_{F}, \ell \times\{p\}\right) .
$$

Since $K_{F}=\Phi^{*} \mathcal{O}_{|E|}(-\operatorname{dim}|E|-1+n) \otimes \Psi^{*}\left(K_{X} \otimes \operatorname{det} E\right)$ and since $\Phi(\ell \times\{p\})=$ $\Phi(\tilde{\ell})=\ell$, it follows from the above inequality that

$$
\left(\Psi^{*}\left(K_{X} \otimes \operatorname{det} E\right), \tilde{\ell}\right) \leq\left(\Psi^{*}\left(K_{X} \otimes \operatorname{det} E\right), \ell \times\{p\}\right) .
$$

By construction, $\Psi(\tilde{\ell})$ is the closure of $\eta_{\{p\}}\left(\left(\ell \cap|E|_{\text {comp }}\right) \times\{p\}\right)$, and hence a (complete) curve by (3), say $C$. On the other hand, $\Psi(\ell \times\{p\})=\{p\}$. Therefore by the above inequality, we have $\left(K_{X} \otimes \operatorname{det} E, C\right) \leq 0$.

Remark 7.2. For a general point $p \in X$ and a general line $\ell \subset\left|E \otimes \mathcal{I}_{\{p\}}\right|$, if we had $(\ell \times\{p\}) \cap B=\emptyset$, then the argument above would be much simpler. But even if $p \in X$ and $\ell \subset\left|E \otimes \mathcal{I}_{\{p\}}\right|$ are general, it can happen that $(\ell \times\{p\}) \cap B \neq \emptyset$ in case (4) in Theorem 1.1.

\section{Proof of the theorem When $\operatorname{sp}(E)>\operatorname{dim} X$}

If the pair $(X, E)$ in Theorem 1.1 has $\operatorname{sp}(E)>n$, by Proposition 7.1, the adjoint bundle $K_{X} \otimes \operatorname{det} E$ is not ample. Hence by Fujita's theorem [6], we have three possibilities in our situation:

(a) $(X, E) \cong\left(\mathbb{P}^{n}, \mathcal{O}_{\mathbb{P}^{n}}(2) \oplus \mathcal{O}_{\mathbb{P}^{n}}(1)^{\oplus n-1}\right)$;

(b) $(X, E) \cong\left(\mathbb{Q}^{n}, \mathcal{O}_{\mathbb{Q}^{n}}(1)^{\oplus n}\right)$;

(c) there exist vector bundles $\mathcal{F}$ and $\mathcal{E}$ of rank $n$ on a smooth projective curve $C$, such that $X$ is isomorphic to a projective space bundle $\mathbb{P}_{C}(\mathcal{F})$ over $C$ with the projection $\pi: \mathbb{P}_{C}(\mathcal{F}) \rightarrow C$ and with the tautological line bundle $\mathcal{O}_{\mathbb{P}_{C}(\mathcal{F})}(1)$, and $E \cong \pi^{*} \mathcal{E} \otimes \mathcal{O}_{\mathbb{P}_{C}(\mathcal{F})}(1)$.

The cases (a) and (b) correspond to (2) and (3) in Theorem 1.1 respectively. Thus we have only to show that the case (c) implies (4) and (5).

First we show that if the vector bundle $E$ in (c) is ample and spanned with $c_{n}(E)=2$ and if $C$ is of genus $g(C) \geq 2$, then $C$ is a hyperelliptic curve with the hyperelliptic pencil $g_{2}^{1}=|\operatorname{det} \mathcal{F} \otimes \operatorname{det} \mathcal{E}|$; this is case (5) in the theorem. Indeed, since $2=c_{n}(E)=\operatorname{deg} \mathcal{F}+\operatorname{deg} \mathcal{E}$ and since $K_{X} \otimes \operatorname{det} E=\pi^{*}\left(K_{C} \otimes \operatorname{det} \mathcal{F} \otimes \operatorname{det} \mathcal{E}\right)$, the adjoint bundle $K_{X} \otimes \operatorname{det} E$ is spanned. Let $g: X \rightarrow \mathbb{P}$ be the morphism associated with the linear system $\left|K_{X} \otimes \operatorname{det} E\right|$ and $h: C \rightarrow \mathbb{P}$ the morphism associated with $\left|K_{C} \otimes \operatorname{det} \mathcal{E} \otimes \operatorname{det} \mathcal{F}\right|$. Then $g=h \circ \pi$. Let $t \in H^{0}(X, E)$ be a general section with $(t)_{0}=\{p, q\}(p \neq q)$. Since $E \mid \pi^{-1}(\pi(p)) \cong \mathcal{O}_{\mathbb{P}^{n-1}}(1)^{\oplus n}$, we have $\pi(p) \neq \pi(q)$. By the Cayley-Bacharach property [8, Proposition 1.22], we have $g(p)=g(q)$, namely, $h(\pi(p))=h(\pi(q))$. Hence the degree of the morphism $C \rightarrow h(C)$ is greater than 1. Let $C^{\prime}$ be the normalization of the image $h(C)$ and $\tilde{h}: C \rightarrow C^{\prime}$ the induced 
morphism. Then $H^{0}\left(C^{\prime}, \mathcal{O}_{C^{\prime}}(1)\right) \cong H^{0}\left(C, K_{C} \otimes \operatorname{det} \mathcal{E} \otimes \operatorname{det} \mathcal{F}\right)$. Thus, for the $\Delta$-genus (see [5]), we have

$$
\begin{aligned}
\Delta\left(C^{\prime}, \mathcal{O}_{C^{\prime}}(1)\right) & =1+\operatorname{deg} \mathcal{O}_{C^{\prime}}(1)-\operatorname{dim}_{k} H^{0}\left(C^{\prime}, \mathcal{O}_{C^{\prime}}(1)\right) \\
& \leq 1+g(C)-(g(C)+1)=0 .
\end{aligned}
$$

Since $\Delta$-genus is nonnegative (see [5]), we have $\Delta\left(C^{\prime}, \mathcal{O}_{C^{\prime}}(1)\right)=0, \operatorname{deg} \tilde{h}=2$, and $\operatorname{deg} \mathcal{O}_{C^{\prime}}(1)=g(C)$. Thus $C^{\prime} \cong \mathbb{P}^{1}$, and hence $C$ is a hyperelliptic curve of genus $g(C) \geq 2$ with the hyperelliptic pencil $g_{2}^{1}=\left|\tilde{h}^{*} \mathcal{O}_{\mathbb{P}^{1}}(1)\right|$. Since $K_{C} \otimes \operatorname{det} \mathcal{F} \otimes \operatorname{det} \mathcal{E} \cong$ $\tilde{h}^{*} \mathcal{O}_{\mathbb{P}^{1}}(g(C))$ and $K_{C} \cong \tilde{h}^{*} \mathcal{O}_{\mathbb{P}^{1}}(g(C)-1)$, we have $\operatorname{det} \mathcal{F} \otimes \operatorname{det} \mathcal{E} \cong \tilde{h}^{*} \mathcal{O}_{\mathbb{P}^{1}}(1)$, i.e., $|\operatorname{det} \mathcal{F} \otimes \operatorname{det} \mathcal{E}|=g_{2}^{1}$.

We look at bundles in the case (c) with $g(C) \leq 1$. When $g(C)=0$ in (c), $C=\mathbb{P}^{1}$ and hence, by Grothendieck's theorem, we may assume that $\mathcal{F}=\mathcal{O}_{\mathbb{P}^{1}}\left(a_{1}\right) \oplus \cdots \oplus$ $\mathcal{O}_{\mathbb{P}^{1}}\left(a_{n}\right)$ and $\mathcal{E}=\mathcal{O}_{\mathbb{P}^{1}}\left(b_{1}\right) \oplus \cdots \oplus \mathcal{O}_{\mathbb{P}^{1}}\left(b_{n}\right)\left(a_{i}, b_{j} \in \mathbb{Z}\right)$. Since $\pi^{*} \mathcal{O}_{\mathbb{P}^{1}}\left(b_{j}\right) \otimes \mathcal{O}_{\mathbb{P}_{\mathbb{P}}(\mathcal{F})}(1)$ $(j=1, \ldots, n)$ is ample as a summand of $E$, the corresponding vector bundle $\mathcal{F} \otimes$ $\mathcal{O}_{\mathbb{P}^{1}}\left(b_{j}\right)$ is ample on $C=\mathbb{P}^{1}$, and hence its summand $\mathcal{O}_{\mathbb{P}^{1}}\left(a_{i}+b_{j}\right)$ is an ample line bundle for every $i=1, \ldots, n$. Thus we have $a_{i}+b_{j} \geq 1$ for every $i, j=1, \ldots, n$ (see also [4, Lemma 3.2.4]). Since $c_{n}(E)=\left(a_{1}+b_{1}\right)+\cdots+\left(a_{n}+b_{n}\right)$, we have $n \leq 2$. So it is easy to see that $(X, E) \cong\left(\mathbb{Q}^{2}, \mathcal{O}_{\mathbb{Q}^{2}}(1)^{\oplus 2}\right)$; this is the case (3).

To look at the case $g(C)=1$, we need the following lemma.

Lemma 8.1. Let $\mathcal{G}$ be a vector bundle of rank $m$ and degree 1 on an elliptic curve C.

(1) When $m \geq 2, \mathcal{G}$ is indecomposable if and only if there is an indecomposable vector bundle $\mathcal{G}^{\prime}$ on $C$ fitting into a nonsplit exact sequence $0 \rightarrow \mathcal{O}_{C} \rightarrow \mathcal{G} \rightarrow$ $\mathcal{G}^{\prime} \rightarrow 0$.

(2) If $\mathcal{G}$ is indecomposable, then $\mathcal{G}$ is ample and $\mathcal{G} \otimes \mathcal{M}$ is ample and spanned for every line bundle $\mathcal{M}$ of degree 1 on $C$.

Proof. (1) See [1, Lemma 15 and Proof of Lemma 16]. (2) The idea and the result in case $m=2$ are in [3]. The ampleness of $\mathcal{G}$ follows from (1) and [7, Theorem 2.2], by induction on $m$. Hence $\mathcal{G} \otimes \mathcal{M}$ is also ample. We prove the spannedness of $\mathcal{G} \otimes \mathcal{M}$ by induction on $m$. We may assume that $\mathcal{M} \cong \mathcal{O}_{C}(y)$ for some $y \in C$. When $m=1$, it is clear. When $m>1$, by (1), we have an exact sequence $0 \rightarrow \mathcal{O}_{C}(y) \rightarrow \mathcal{G} \otimes \mathcal{O}_{C}(y) \rightarrow \mathcal{G}^{\prime} \otimes \mathcal{O}_{C}(y) \rightarrow 0$. Since $h^{1}\left(C, \mathcal{O}_{C}(y)\right)=0$ and $\mathcal{G}^{\prime} \otimes \mathcal{O}_{C}(y)$ is spanned by the induction assumption, $\mathcal{G} \otimes \mathcal{O}_{C}(y)$ is spanned at every point but $\{y\}$. Applying this argument for $\mathcal{G} \otimes \mathcal{O}_{C}(y-x)$ and $\mathcal{O}_{C}(x)$ for some $x \neq y \in C$, instead of $\mathcal{G}$ and $\mathcal{M}$, we see that $\mathcal{G} \otimes \mathcal{O}_{C}(y)$ is also spanned at $\{y\}$.

Now we go back to the proof of Theorem 1.1. The case remaining is (c) for a smooth curve $C$ with $g(C)=1$. Recall that for a vector bundle $\mathcal{G}$ on $C$, a line subbundle $\mathcal{B}$ of $\mathcal{G}$ is said to be maximal if $\operatorname{deg}(\mathcal{B})=\max \{\operatorname{deg} \mathcal{N} ; \mathcal{N}$ is a line subbundle of $\mathcal{G}\}$ (see [12]). We take line bundles $\mathcal{L}_{n}, \ldots, \mathcal{L}_{1}$ and $\mathcal{M}_{n}, \ldots, \mathcal{M}_{1}$ on $C$ so that $\mathcal{L}_{i}\left(\right.$ resp. $\mathcal{M}_{i}$ ) is a maximal line subbundle of $\mathcal{F}_{i}\left(\right.$ resp. $\left.\mathcal{E}_{i}\right)$ with $\mathcal{F}_{n}:=\mathcal{F}$ (resp. $\mathcal{E}_{n}:=\mathcal{E}$ ) and $\mathcal{F}_{i-1}:=\mathcal{F}_{i} / \mathcal{L}_{i}\left(\right.$ resp. $\left.\mathcal{E}_{i-1}:=\mathcal{E}_{i} / \mathcal{M}_{i}\right)$ for $i=n-1, \ldots, 1$. If we set $\mathcal{A}=\mathcal{L}_{n} \otimes \mathcal{M}_{n}, \mathcal{F}^{\prime}=\mathcal{F} \otimes \mathcal{L}_{n}^{-1}$, and $\mathcal{E}^{\prime}=\mathcal{E} \otimes \mathcal{M}_{n}^{-1}$, then we have $X \cong \mathbb{P}_{C}\left(\mathcal{F}^{\prime}\right)$ and $E \cong \pi^{*}\left(\mathcal{E}^{\prime} \otimes \mathcal{A}\right) \otimes \mathcal{O}_{\mathbb{P}_{C}\left(\mathcal{F}^{\prime}\right)}(1)$ for the tautological line bundle $\mathcal{O}_{\mathbb{P}_{C}\left(\mathcal{F}^{\prime}\right)}(1)$ of $\mathbb{P}_{C}\left(\mathcal{F}^{\prime}\right)$. Thus we may assume that $(X, E)=\left(\mathbb{P}_{C}(\mathcal{F}), \pi^{*}(\mathcal{E} \otimes \mathcal{A}) \otimes \mathcal{O}_{\mathbb{P}_{C}(\mathcal{F})}(1)\right)$ for a line bundle $\mathcal{A}$ on $C$ and for vector bundles $\mathcal{F}$ and $\mathcal{E}$ of rank $n$ on $C$ with $\mathcal{L}_{n}=\mathcal{O}_{C}$ and $\mathcal{M}_{n}=\mathcal{O}_{C}$. 
With this notation, it follows from [12] that

$$
\operatorname{deg} \mathcal{F}_{i}-i \cdot \operatorname{deg} \mathcal{L}_{i} \leq(i-1) g(C)=i-1, \quad \text { and } \quad \operatorname{deg} \mathcal{E}_{i}-i \cdot \operatorname{deg} \mathcal{M}_{i} \leq i-1
$$

for each $i=1, \ldots, n$. By descending induction on $i$, we get $\operatorname{deg} \mathcal{F}_{i} \leq i$ and $\operatorname{deg} \mathcal{E}_{i} \leq$ $i$. In particular, we have

$$
\operatorname{deg} \mathcal{L}_{1} \leq 1, \quad \text { and } \quad \operatorname{deg} \mathcal{M}_{1} \leq 1 .
$$

Since $E^{\prime}:=E \mid \mathbb{P}_{C}\left(\mathcal{F}_{n-1}\right)$ is ample and spanned with

$$
c_{n-1}\left(E^{\prime}\right)=\operatorname{deg} \mathcal{F}_{n-1}+(n-1) \operatorname{deg} \mathcal{A}+\operatorname{deg} \mathcal{E}_{n-1} \geq 2
$$

by [15, Theorem 3.4], comparing $c_{n}(E)=\operatorname{deg} \mathcal{F}+n \operatorname{deg} \mathcal{A}+\operatorname{deg} \mathcal{E}=2$ with $c_{n-1}\left(E^{\prime}\right)$, we have

$$
\operatorname{deg} \mathcal{A} \leq 0
$$

On the other hand, since $\mathcal{E}_{i} \otimes \mathcal{A} \otimes \mathcal{L}_{1} \cong \pi^{*}\left(\mathcal{E}_{i} \otimes \mathcal{A}\right) \otimes \mathcal{O}_{\mathbb{P}_{C}(\mathcal{F})}(1) \mid \mathbb{P}\left(\mathcal{L}_{1}\right)$ is ample and spanned, the dimension of the vector space of its global sections is greater than the rank of $\mathcal{E}_{i} \otimes \mathcal{A} \otimes \mathcal{L}_{1}$, and hence the bundle $\mathcal{E}_{i} \otimes \mathcal{A} \otimes \mathcal{L}_{1} \otimes \mathcal{O}_{C}(-x) \cong$ $\left(\mathcal{E}_{i} \otimes \mathcal{M}_{i}^{-1}\right) \otimes \mathcal{M}_{i} \otimes \mathcal{A} \otimes \mathcal{L}_{1} \otimes \mathcal{O}_{C}(-x)$ has a non-zero global section for any $x \in C$. Therefore the degree of $\mathcal{M}_{i} \otimes \mathcal{A} \otimes \mathcal{L}_{1} \otimes \mathcal{O}_{C}(-x)$ is nonnegative, since $\mathcal{M}_{i}$ is a maximal line subbundle of $\mathcal{E}_{i}$. The same is true for $\mathcal{F}_{i} \otimes \mathcal{A} \otimes \mathcal{M}_{1}$ and $\mathcal{L}_{i} \otimes \mathcal{A} \otimes \mathcal{M}_{1} \otimes \mathcal{O}_{C}(-x)$. Thus for each $i=2, \ldots, n$, we have

$$
\operatorname{deg} \mathcal{M}_{i}+\operatorname{deg} \mathcal{A}+\operatorname{deg} \mathcal{L}_{1} \geq 1, \quad \text { and } \quad \operatorname{deg} \mathcal{L}_{i}+\operatorname{deg} \mathcal{A}+\operatorname{deg} \mathcal{M}_{1} \geq 1 .
$$

Since $\mathcal{L}_{n}=\mathcal{M}_{n}=\mathcal{O}_{C}$, from (8.2), (8.3), and (8.4) with $i=n$, it follows that $\operatorname{deg} \mathcal{A}=0$ and $\operatorname{deg} \mathcal{L}_{1}=\operatorname{deg} \mathcal{M}_{1}=1$. Hence, from (8.4) and $\operatorname{deg} \mathcal{F}+n \operatorname{deg} \mathcal{A}+$ $\operatorname{deg} \mathcal{E}=2$, it follows that $\operatorname{deg} \mathcal{L}_{i}=\operatorname{deg} \mathcal{M}_{i}=0$ for $i=2, \ldots, n-1$.

Then the exact sequences

$$
0 \rightarrow \mathcal{L}_{i} \rightarrow \mathcal{F}_{i} \rightarrow \mathcal{F}_{i-1} \rightarrow 0 \quad \text { and } \quad 0 \rightarrow \mathcal{M}_{i} \rightarrow \mathcal{E}_{i} \rightarrow \mathcal{E}_{i-1} \rightarrow 0
$$

are nonsplit for each $i=1, \ldots, n$, since $\mathcal{E}_{i} \otimes \mathcal{A} \otimes \mathcal{L}_{1}$ and $\mathcal{A} \otimes \mathcal{M}_{1} \otimes \mathcal{F}_{i}$ are ample and spanned. Hence $\mathcal{F}$ and $\mathcal{G}$ are indecomposable by Lemma 8.1 (1). By replacing $\mathcal{E}$ by $\mathcal{E} \otimes \mathcal{A}$ and $\mathcal{A}$ by $\mathcal{O}_{C}$, it turns out that $(X, E)$ is of case (4).

\section{Ampleness, SPANNEDNESS AND $c_{2}(E)=2$}

Conversely, we check that $(X, E)$ in (1), (2), (3) and (4) of Theorem 1.1 are ample and spanned, with $c_{n}(E)=2$. It is only nontrivial that $E$ in (4) is ample and spanned. Since $\mathcal{F}$ and $\mathcal{E}$ are indecomposable of degree 1 on an elliptic curve $C$, by Lemma $8.1(1)$, there exist vector bundles $\mathcal{F}_{i}$ and $\mathcal{E}_{i}, \operatorname{rank} \mathcal{F}_{i}=\operatorname{rank} \mathcal{E}_{i}=i$ $(i=1, \ldots, n)$, with $\mathcal{F}_{n}=\mathcal{F}$ and $\mathcal{E}_{n}=\mathcal{E}$, such that

$$
0 \rightarrow \mathcal{O}_{C} \rightarrow \mathcal{F}_{i} \rightarrow \mathcal{F}_{i-1} \rightarrow 0 \quad \text { and } \quad 0 \rightarrow \mathcal{O}_{C} \rightarrow \mathcal{E}_{i} \rightarrow \mathcal{E}_{i-1} \rightarrow 0
$$

are nonsplit exact sequences. By Lemma $8.1(2), \mathcal{O}_{\mathbb{P}_{C}(\mathcal{F})}(1)$ is ample on $X=\mathbb{P}_{C}(\mathcal{F})$ and hence so is $E$. For the spannedness of $E$, we show that $E_{m}:=\pi^{*} \mathcal{E}_{m} \otimes$ $\mathcal{O}_{\mathbb{P}_{C}\left(\mathcal{F}_{n}\right)}(1)$ is spanned, by induction on $n=\operatorname{dim} X$ and $m=\operatorname{rank} E_{m}$. By Lemma $8.1(2)$, it is clear when $n=1$ or when $n \geq 2$ and $m=1$. When $n \geq 2$ and $m \geq 2$, since $0 \rightarrow \mathcal{O}_{\mathbb{P}_{C}\left(\mathcal{F}_{n}\right)}(1) \rightarrow E_{m} \rightarrow E_{m-1} \rightarrow 0$ is exact and $h^{1}\left(X, \mathcal{O}_{\mathbb{P}_{C}\left(\mathcal{F}_{n}\right)}(1)\right)=$ $h^{1}\left(C, \mathcal{F}_{n}\right)=0, E_{m}$ is spanned outside of $\mathbb{P}_{C}\left(\mathcal{F}_{n-1}\right) \in\left|\mathcal{O}_{\mathbb{P}_{C}\left(\mathcal{F}_{n}\right)}(1)\right|$ by the induction assumption on $m$. Since $H^{0}\left(X, E_{m}\right) \rightarrow H^{0}\left(X, E_{m} \mid \mathbb{P}_{C}\left(\mathcal{F}_{n-1}\right)\right)$ is surjective by $h^{1}\left(X, \pi^{*} \mathcal{E}_{m}\right)=h^{1}\left(C, \mathcal{E}_{m}\right)=0$, the induction assumption on $n$ implies that $E_{m}$ is also spanned on $\mathbb{P}_{C}\left(\mathcal{F}_{n-1}\right)$. This completes the proof. 


\section{REFERENCES}

1. M. F. Atiyah, Vector bundles over an elliptic curve, Proc. London Math. Soc. (3) 7 (1957), 414-452. MR 24:A1274

2. E. Ballico, Rank-2 vector bundles with many sections and low $c_{2}$ on a surface, Geom. Dedicata 29 (1989), 109-124. MR 90e:14014

3. E. Ballico and A. Lanteri, An indecomposable rank-2 vector bundle the complete linear system of whose determinant consists of hyperelliptic curves, Boll. Un. Mat. Ital. (7) 3-A (1989), 225-230. MR 90j:14022

4. M. C. Beltrametti and A. J. Sommese, The adjunction theory of complex projective varieties, de Gruyter Exp. Math. 16, de Gruyter, Berlin, 1995. MR 96f:14004

5. T. Fujita, Classification theories of polarized varieties, London Math. Soc. Lecture Note Ser. Vol. 155, 1990. MR 93e:14009

6. T. Fujita, On adjoint bundles of ample vector bundles, Complex Algebraic Varieties, Proceedings, Bayreuth 1990 (K. Hulek et al., eds.), Lecture Notes in Math., vol. 1507, Springer-Verlag, 1992, pp. 105-112. MR 93j:14052

7. D. Gieseker, p-ample bundles and their Chern classes, Nagoya Math. J. 43 (1971), 91-116. MR 45:5139

8. P. Griffiths and J. Harris, Residues and zero-cycles on algebraic varieties, Ann. of Math. 108 (1978), 461-505. MR 80d:14006

9. Y. Kawamata, The cone of curves of algebraic varieties, Ann. of Math. 119 (1984), 603-633. MR 86c: $14013 \mathrm{~b}$

10. A. Lanteri and F. Russo, A footnote to a paper by Noma, Rend. Mat. Acc. Lincei (9) 4 (1993), 131-132. MR 95a:14045

11. A. Lanteri and A. J. Sommese, A vector bundle characterization of $\mathbb{P}^{n}$, Abh. Math. Sem. Univ. Hamburg 58 (1988), 89-94. MR 91e:14054

12. S. Mukai and F. Sakai, Maximal subbundles of vector bundles on a curve, Manuscripta Math. 52 (1985), 251-256. MR 86k:14013

13. A. Noma, Classification of rank-two ample and spanned vector bundles on surfaces whose zero loci consist of general points, Transactions Amer. Math. Soc. 342 (1994), 867-894. MR 89f: 14040

14. A. Noma, Ample and spanned vector bundles of $c_{2}=2$ on normal Gorenstein surfaces, preprint.

15. A. Wiśniewski, Length of extremal rays and generalized adjunction, Math. Z. 200 (1989), 409-427. MR 91e:14032

16. Q. Zhang, Ample vector bundles on singular varieties, Math. Z. 220 (1995), 59-64. MR 96i: 14034

17. Q. Zhang, Ample vector bundles on singular varieties II, Math. Ann. 307 (1997), 505-509. CMP 97:09

Department of Mathematics, Faculty of Education, Yokohama National University, 156 Tokimadai, Hodogaya, Yokohama 240, Japan

E-mail address: noma@ms.ed.ynu.ac.jp 